\title{
INDUCED HYPOTENSIVE ANESTHESIA DURING ELECTIVE SURGICAL PROCEDURES
}

Baljit Singh Bajwa ${ }^{1}$

\section{HOW TO CITE THIS ARTICLE:}

Baljit Singh Bajwa. "Induced Hypotensive Anesthesia during Elective Surgical Procedures". Journal of Evolution of Medical and Dental Sciences 2014; Vol. 3, Issue 30, July 28; Page: 8418-8426,

DOI: $10.14260 /$ jemds/2014/3064

\begin{abstract}
Medical paradox of induced hypotension has arisen because bleeding during operative interventions has become a problem of increasing importance during the past few years. Certain surgical procedures are now so vastly extensive and that other operations have penetrated so deeply into the vital structure of the body. A number of workers, both on this continent and abroad, have suggested that one effective method of controlling either gross hemorrhage or excessive vascular ooze may be by the reduction of systemic arterial pressure. When such a deliberate hypotension is combined with proper posturing of the patient so that the operative site is superior, permitting gravitational venous drainage away from the wound to the dependent portions of the body, the technique has been called "controlled hypotension," and has been employed to achieve a relatively ischemic operative field.
\end{abstract}

KEYWORDS: Hypotensive anesthesia, bleeding, hemodynamics, metabolism.

INTRODUCTION: For half a century, controlled hypotension has been used to reduce the bleeding and the need for blood transfusion, and provide a satisfactory bloodless surgical field. It has been indicated in oromaxillofacial surgery (mandibular osteotomy, facial repair), endoscopic sinus or middle ear microsurgery, spinal surgery and other neurosurgery (aneurysm), major orthopedic surgery (hip or knee replacement, spinal), prostatectomy, cardiovascular surgery and liver transplant surgery. Controlled hypotension defined as a reduction of the systolic blood pressure to $80-90 \mathrm{~mm}$ $\mathrm{Hg}$, a reduction of mean arterial pressure (MAP) to $50-60 \mathrm{~mm} \mathrm{Hg}$ or a $30 \%$ reduction of baseline MAP.

The greatest efficacy and ease-of-use to toxicity ratio is for techniques of anesthesia that associate analgesia and hypotension at clinical concentrations without the need for potent hypotensive agents.

It is recommended that Hypotensive anesthesia be adjusted in relation to the patient's preoperative blood pressure rather than a specific target pressure and be limited to that level necessary to reduce bleeding in the surgical field and in duration to that part of the surgical procedure deemed to benefit by it.

Appropriate patient evaluation and selection, proper positioning and monitoring, and adequate flood therapy are stressed as important consideration in patients undergoing induced hypotension during surgery.

The aim of this article to view general factors that influence surgical bleeding and to discuss measures for reducing it.

PHYSIOLOGY: In order to use induced hypotension for the optimal benefit of the patient, it is necessary to have an understanding of the regulation of blood flow of vital organs. Even relatively 


\section{REVIEW ARTICLE}

short periods of unwanted hypotension, such as those associated with "shock," may be followed by irreversible organ damage. In this situation, the severe decrease in organ blood flow is accompanied by acid-based and metabolic changes. Controlled hypotension rarely results in damage, however, because organ blood flow is normally well maintained.

Richly perfused tissues, such as the brain, heart, liver and kidneys exhibit autoregulation of their own blood supply through mechanisms related to the intrinsic elasticity of the vascular smooth muscle and vasodilator substances produced in the metabolically active tissues. That is, they maintain their perfusion over a wide range of pressure changes, and it is only when the pressure decreases to relatively low value that adequate perfusion cannot be maintained. This critical pressure varies from vessel to vessel, organ to organ, and probably from individual to individual.

Normally, cerebral blood flow remains constant over a mean arterial pressure (MAP) range of 60 to $150 \mathrm{~mm} \mathrm{Hg}{ }^{1}$

Volatile anesthetic drugs attenuate or abolish the autoregulation of cerebral blood flow in a dose-dependent manner in the following order: halothane $>$ enflurane $>$ isoflurane. ${ }^{2}$ The relative potency of these agents to decrease tissue metabolism is in the reverse order. ${ }^{3}$ Decreases in the arterial carbon dioxide tension $\left(\mathrm{PaCO}_{2}\right)$ causes cerebral vasoconstriction. For every millimeter decrease in $\mathrm{PaCO}_{2}$, the cerebral blood flow decreases by $2 \%$ in normotensive subjects. ${ }^{4}$

This effect is either updated 5 or abolished 6,7during hypotension, depending upon the hypotensive agent. With isoflurane, the decrease in cerebral blood flow appears to be less than or equal to decrements in cerebral oxygen demand regardless of whether the patient is hypocapnic. ${ }^{8}$

Vasodilators such as sodium nitroprusside and nitroylcerin attenuate the autoregulation of blood flow in a in a similar manner to that the volatile agents. ${ }^{3}$

If the reduction in cerebral blood flow exceeds the reduction in cerebral metabolic requirement for oxygen, cerebral ischemia may develop. When cerebral blood flow is reduced by $40 \%$ to $50 \%$ below control values, ischemic changes appear in the electroencephalogram (EEG), and below $60 \%$ the EEG become iso-electric. ${ }^{9}$

Elevation of the head during hypotensive anesthesia can aggravate the decrease in cerebral perfusion pressure. The perfusion pressure decreases by $2 \mathrm{~mm} \mathrm{Hg}$ for every $2.5 \mathrm{~cm}$ the head is raised above the point of monitoring. ${ }^{10,11}$

A head-up position in a patient decreases the apical perfusion of the lungs due to gravity, thus increasing dead space. ${ }^{11}$ Accordingly, the $\mathrm{PaCO}_{2}$ may rise. Induced hypotension may also decrease pulmonary artery pressure, increase blood flow through dependent regions of the lungs, and inhibit the hypoxic pulmonary vasoconstrictive effect, thereby increasing the intrapulmonary shunt and the $\mathrm{PaCO}_{2}{ }^{13}$ This inhibition occurs with nitroprusside, nitroglycerin, and calcium-channel blockers, and to a lesser extent with isoflurane. ${ }^{14,15}$

The arterial oxygen tension $\left(\mathrm{PaO}_{2}\right)$ may be reduced during hypotension for the same reason described for the $\mathrm{PaCO}_{2}$. Thus, it is essential to maintain the oxygen saturation within normal limits, which may necessitate giving more than the normal amount of oxygen during hypotensive anesthesia. However, 100\% oxygen is not preferable during hypotension, as this may cause vasoconstriction, especially in the brain.

Even at rest, cardiac muscle extracts most of the oxygen delivered to it. Consequently, any decrease in myocardial oxygen demand requires a parallel increase in coronary artery blood flow. Hypotensive anesthesia may substantially decrease coronary blood flow. However it simultaneously 
decreases myocardial oxygen demand, due to the reduction in afterload (usually) and/or preload (sometimes). Furthermore, the coronary arteries dilate in response to the accumulation of metabolic vasodilator substances in the myocardium, thus ensuring an educate blood flow to the myocardium. Hypotension with a normal or slow pulse is more beneficial than one with tachycardia. Tachycardia will increase the oxygen demand and shorten diastole, thus decreasing blood flow to the left ventricular subendocardium.

Coronary blood flow is rarely compromised in pediatric patients. ${ }^{9}$ Factors that increase myocardial oxygen demand, such as tachycardia, should rarely cause myocardial ischemia even when the child is hypotensive. In patients with coronary artery obstruction, vasodilators used for hypotension may induce unfavorable intramyocardial blood flow redistribution as a consequence of vessel dilatation, resulting in regional poststenotic hypoperfusion (the "steal" phenomenon) and possible myocardial ischemia. ${ }^{16}$

Decrease in renal blood flow stimulates the rennin-angiotensin system. When the MAP falls below $75 \mathrm{~mm} \mathrm{Hg}$, the glomerular filtration rate falls. ${ }^{17}$ Opioid analgesics and probably most inhalational agents stimulate secretion of antidiuretic hormone. ${ }^{18}$ All these factors result in oliguria during hypotensive anesthesia. However following termination of hypotensive anesthesia, urine formation rapidly recovers, provided the patient is well hydrated. ${ }^{19}$

Since the aim of hypotensive anesthesia is to reduce blood loss and provide easily visualizes surgical field, the degree of reduction should depend on each individual patient and clinical situation. The hypotension should be considered satisfactory when bleeding appears to be minimal and organ perfusion adequate.

CONTRAINDICATIONS: Contraindications to the use of hypotensive anesthesia are listed in Table1. Most contraindications are relative, depending on the type of surgery, the individual patient, the knowledge base and skill level of the anesthetist, and the drug or technique to be used. Absolute contraindications include known drug allergy, inability to monitor the patient adequately, and unfamiliarity with the technique.

Table 1: Contraindications to the use of Hypotensive Anesthesia:

\begin{tabular}{|l|}
\hline Anesthetist Limitations \\
Lack of understanding of the technique \\
Lack of technical experience \\
Inability to monitor the patient adequately \\
Patient limitations \\
Cardiac disease \\
Diabetes mellitus \\
Gross anemia, hemoglobinopathies, polycythemia \\
Hepatic disease \\
Intolerance to the drugs available to produce hypotension \\
Ischemic cerebrovascular disease \\
Renal disease \\
Respiratory insufficiency \\
Severe systemic hypotension
\end{tabular}


Cardiovascular System: Mild coronary Ischemia may occur during induced systolic hypotension below $60 \mathrm{~mm} \mathrm{Hg.20}$ Patients with a previous history of hypertension are more susceptible to Ischemia. Furthermore, rapid hypotension may be accompanied by an earlier onset of Ischemic changes in the electrocardiogram (ECG). ${ }^{21}$

Skin Necrosis: Necrosis of the skin with ulcer formation can occur after prolonged hypotensive anesthesia from pressure-induced hypoperfusion. Common sites are the tip of nose (due to the pressure of the endotracheal tube or nasogastric tube) and pressure areas in the sacral region with the patient in the reverse Trendelenberg position. Recently, there was a report ${ }^{22}$ of a finger injury from pressure of a finger probe (pulse oximeter) in a patient who had received hypotensive anesthesia. In a similar manner, thermal burns may appear in sacral areas when patients are placed on warming blankets during hypotensive anesthesia. ${ }^{23}$

Cyanide Poisoning: Large dose of nitroprusside administered to effect hypotension can result in cyanide poisoning, which manifests as metabolic acidosis, persistent hypotension, and tachycardia. ${ }^{24-28}$ In some cases it has been fatal. ${ }^{26-28}$

\section{Mechanical Maneuvers to Potentiate the Action of Hypotensive Agents:}

Positioning: The importance of correct positioning of the patient during controlled hypotension in anesthesia cannot be overemphasized ${ }^{11-12}$. Elevation of the site of operation allows easy drainage of venous blood from the site of surgery. Inclining the patient (raising the head) $15^{\circ}$ to $25^{\circ}$ off the horizontal plane facilitates pooling of blood in the dependent regions of the body, thus reducing venous return to the heart and the cardiac preload. Blood pressure is also reduced gravimetrically in the operating field. For each $2.5-\mathrm{cm}$ elevation of the site above the heart, there is a $2-\mathrm{mm} \mathrm{Hg}$ fall in blood pressure. The hypotensive effects of most of the hypotensive agents are potentiated by posture. Rapid changes in posture may be followed by rapid changes in blood pressure; thus, tilting the head down may be used to rapidly reverse excessive hypotension.

Positive Airway Pressure: Use of positive pressure ventilation decreases the venous return during the positive pressure phase. ${ }^{34}$ This effect can be enhanced by increasing the inspiratory volume, prolonging the inspiratory time, and raising the positive end-expiratory pressure. ${ }^{31,35}$ However, these manipulations not only cause a deleterious effect on the cardiac output and pulmonary perfusion, but also result in increased respiratory dead space. Increasing intrathoracic pressure can also decrease venous outflow from the cranium sufficiently to increase the cerebral blood volume and intracranial pressure. Thus, respirator manipulations to minimize venous return are usually not employed in controlled hypotension.

\section{ANESTHETIC MANAGEMENT:}

Preoperative Management: The decision to induce hypotension is best taken preoperatively following examination of the patient. As a crucial prerequisite, the anesthetist conducting the hypotensive anesthesia must have a thorough knowledge of the technique that is to be performed. Appropriate patient evaluation and selection are also important for safe induced hypotensive anesthesia. Preoperative investigations including hemoglobin $(\mathrm{Hb})$, urea, electrolytes, and ECG 


\section{REVIEW ARTICLE}

should be carried out. A minimum $\mathrm{Hb}$ of $10 \mathrm{~g} / \mathrm{dL}$ should be safe in those undergoing hypotensive anesthesia. Either in the ward before transport or in the operating room before anesthesia, arterial blood gas sampling may help provide a baseline for intraoperative or postoperative measurements. Various premedications, including anxiolytics, analgesics, a blockers (eg, droperidol, chlorpromazine), b blockers (eg, metoprolol), ${ }^{33}$ and antihypertensives (eg, clonidine), ${ }^{32,36}$ can assist the induction of hypotension during anesthesia. Vagolytic drugs are best avoided.

Intraoperative Management: Following preanesthetic monitoring, induction of anesthesia can be carried out in the usual manner. A stress free induction and obtunding the increase in blood pressure and tachycardia to intubation will help set the stage for a smooth hypotensive technique. The endotracheal tube and any other tube placed through the nares should not exert undue pressure on the tip of the nose. A second intravenous line is preferable if the hypotensive drug is to be given as an intravenous infusion. During maintenance of anesthesia, patient who is to undergo hypotensive anesthesia during orthognathic surgery is usually ventilated with the help of neuromuscular blocking agents.

Posture: As mentioned previously, the patient should be positioned with the head elevated about $20^{\circ}$ above the heart. Having the legs slightly elevated by flexing the table at the hips of the patient prevents the patient slipping down the table and prevents pressure sores in the sacral region during hypotensive anesthesia. Soft cotton wool pads for the sacral region and the feet, the regions which act as pressure points, will help to prevent skin necrosis.

Monitoring: Meticulous monitoring is essential for patient safety during hypotensive anesthesia. Blood pressure, intraarterial blood pressure monitoring during hypotensive anesthesia is recommended by many as it not only helps in getting immediate and often more accurate blood pressure data than can be obtained with noninvasive blood pressure monitors but also permits sampling for arterial blood gases, hematocrit, and other laboratory investigations.

If noninvasive blood pressure monitoring is also to be used, attaching a blood pressure cuff to the same arm that is used for arterial cannulation will help check the accuracy of blood pressure recordings from the indwelling catheter. Noninvasive monitoring will also be helpful during periods of problems with arterial blood pressure monitoring. Although not the ideal, hypotensive anesthesia may be carried out without invasive blood pressure monitoring, especially when the procedure is short and techniques are used that are not likely to cause rapid swings in blood pressure.

Electrocardiogram: Monitoring of the $\mathrm{V}_{5}$ lead of the ECG with an appropriate input filter, which is essential for ST-segment analysis, may give an indication of cardiac Ischemia if it occurs and warn the anesthetist that the coronary blood supply is compromised.

Oxygen saturation: It is important to monitor oxygen saturation, as hypotension can cause a mismatch of ventilation and perfusion in the lungs as well as reduced blood flow to peripheral tissues. The pulse oximeter probe should not exert undue pressure on the finger tip. 
End-tidal carbon dioxide: The measurement of the end-tidal carbon dioxide tension $\left(\mathrm{EtCO}_{2}\right)$ will give an indication of the $\mathrm{PaCO}_{2}$ and will help prevent both hypo and hypercarbia. However, the relationship between $\mathrm{PaCO} 2$ and EtCO2 changes with hypotension, so that arterial blood gas determinations also should be carried out intermittently to make sure that the $\mathrm{PaCO} 2$ is within the desired range.

Temperature: Core temperature monitoring is important during controlled hypotension, because body heat dissipates more rapidly from dilated vessels. Lowered temperature may decrease the effectiveness of vasodilators and increase the dose requirements if compensatory vasoconstriction occurs.

Central venous pressure: A greater hypotensive response will be exhibited by hypovolemic patients at the onset of controlled hypotension. Central venous pressure measurements will help in determining the adequacy of circulating blood volume at the start of hypotension and will help to maintain it during the period of controlled hypotension.

Urine Volume: Urine output decreases during hypotensive anesthesia. However, some urine is formed. If the operation is to exceed $5 \mathrm{hrs}$. a urinary catheter should be inserted. By measuring the urine output, it is possible to ensure that renal perfusion is maintained. Usually, once the hypotension is reversed, during output reverts to normal.

Blood loss, the physiological response to blood loss may be lost during hypotensive anesthesia. Therefore, blood loss should be carefully estimated using swab weighing and measuring blood volume in suction bottles.

Cardiac output: The decrease in cardiac output during hypotensive anesthesia can be measured by the insertion of pulmonary artery catheters. The catheters can also help in monitoring the fluid status, left heart function, and mixed venous oxygen content.

Fluid Therapy: Proper fluid therapy is essential during hypotensive anesthesia. The aim of induced hypotension is to lower the MAP while maintaining adequate perfusion of all vital organs. Thus, preoperative fluid and electrolyte status must be assessed and corrected if necessary before anesthesia. During anesthesia, the fluid deficit acquired in the preoperative period because of fasting should be calculated and replaced during the first 1 or $2 \mathrm{hr}$. For maintenance, 5 to $6 \mathrm{~mL} / \mathrm{kg}$-hr. of crystalloids should be infused.

Blood loss must be carefully observed, and replacement carried out with either an equal amount of colloid or three times the amount in crystalloid. If the blood loss exceeds $20 \%$ to $25 \%$ of the patient's total blood volume, the loss should be replaced with blood. In operations where major blood loss is expected, it is preferable to insert a central venous catheter to ensure adequacy of the circulating volume.

Induction of hypotension should begin at the time of mucosal incision. That will give time to attain the required MAP before osteotomy. Once hypotension is induced, the required level of blood pressure to minimize blood loss may be maintained by adjusting the amount of hypotensive agent, either manually or automatically by self-tuning adaptive control.29, 30 
Hypotension should be carried out only to that level needed to reduce bleeding and only for that time of the surgery where it is of benefit in reducing significant blood loss.

Postoperative Management: After anesthesia with induced hypotension, adequate postoperative care with resuscitation facilities and experienced nursing staff is essential to prevent morbidity and/or mortality that can occur during this period. Postoperatively, attention should be given to airway maintenance, oxygenation, analgesia, monitoring, positioning, reactionary hemorrhage, and fluid balance. Persistent hypotension, which may be related to the positioning of the patient, a prolonged action of the hypotensive drugs, or both, may occur. Rebound hypertension, especially with inadequate analgesia, is also a concern during this period.

CONCLUSION: Hypotensive anesthesia is undoubtedly of great value in improving the quality of the surgical field. Although autologous blood transfusion and normovolemic hemodilution may be acceptable alternatives to homologous blood transfusion, but they do not constitute a universal replacement for homologous blood transfusion ${ }^{37}$ reverse trendelenberg position, surgical site packing, keeping only the current site of operation exposed at one time, injection of local anesthesia with epinephrine, and meticulous surgical technique with gentle tissue manipulation and use of cautery and ties are additional methods that help in reducing blood loss.

\section{BIBLIOGRAPHY:}

1. Lesssen NA. Cerebral blood flow and oxygen uptake. Physiol Rev 1959; 39: 183-238.

2. Shapiro H. Cerebral blood flow and anesthetics.in; Annual Refresher Course Lecture. American Society of Anesthesiologists 1988; 154: 1.

3. Harp JR, Wollman H. Cerebral metabolic effects of hyperventilation and deliberate hypotension. Br J Anaesth 1973; 45; 256-262.

4. Artru AA. Partial Preservation of Cerebral vascular responsiveness to hypocapnia during isoflurane induced hypotension in dogs. Anesth Analg 1986; 65; 660-666.

5. Artru AA. Cerebral Vascular response to hypocapnia during nitroglycerine-induced hypotension. Neurosurgery 1985; 16: 468-472.

6. Artru AA, Colley PS. Cerebral blood flow response to hypocapnia during hypotension. Stroke 1984; 15: 878-883.

7. Newman B, Gelb AW Lam AM. The Effect of isoflurane -induced hypotension on cerebral blood flow and cerebral metabolic rate for oxygen in humans. Anesthesiology1986; 64: 307-310.

8. Ishikawa T, Funatsu N, Okamoto K, Takishita H McDowel DG. Blood brain barrier function following drug induced hypotension in the dog. Anesthesiology 1983; 59: 526-531.

9. Lermon J. Controlled hypotension. In: Gregory GA, ed: Pediatric Anesthesia, $2^{\text {nd }}$ ed. New York, Churchill living stone, 1989.

10. Enderby GEH. Postural ischaemia and Blood - pressure. Lancet 1954;1:185-187

11. Enderby GEH. Some observation on the practice of deliberate hypotension. Br J 1975; 47; 743744.

12. Eckenhoff JE, Enderby GEH, Larson A, Edridge A, Judevine DE. Pulmonary gas exchange during deliberate hypotension. Br J Anaesth 1963; 35:750-759. 


\section{REVIEW ARTICLE}

13. Cashthely PA, Lear S Cottrell JE, Lear E. Intrapulomonary shunting during induced hypotension. Anesth Analg 1982; 61: 231-235.

14. Hales c, Slate J, Westphal D. Blockade of alveolar hypoxic pulmonary vasoconstriction by sodium nitroprusside and nitroglycerin. Am Rev Respir Dis 1978; 115: 335.

15. Carlsson AJ, Bindslev L, Hedentierna G. Hypoxia induced pulmonary vasoconstriction in the human lung: the effect of isoflurane anesthesia. Anesthesiology 1987; 66: 312-316.

16. Petrozza PH. Induced hypotension. Int Anesthesiol Clin1990; 28: 223-229.

17. Ganong WF. Review of Medical Physiology. Norwalk, Appleton and Lange, 1989:600.

18. Strun L. Organ perfusion during controlled hypotension. Br J Anaesth 1975; 47:793-798.

19. McDougal WS. Renal perfusion/reperfusion injuries. J Urol 1988; 140: 1325-1330.

20. Rollason WN, Hough JM. A study of hypotensive anaesthesia in the elderly. Br J Anaesth 1960; 32:276-285.

21. Rollason WN, Hough JM. A re-examination of some electrocardiographic studies during hypotensive anaesth. Br J Anaesth 1969; 41: 985-986.

22. Chemello PD, Nelson SR, Wolford LM. Finger injury resulting from pulse oximeter probe during orthognatic surgery. Oral Surg Oral Med Oral Pathol 1990; 69: 161-163.

23. Warner WA, Shumrick DA, Caffrey JA. Clinical investigation of prolonged induced hypotensive in head and neck surgery. Br J Anaesth 1970; 42: 39-44.

24. Persheau RA, Modell JH, Briight RN, Shirley PD. Suspected sodium nitroprusside induced cyanide intoxication. Anaesth Analog 1977; 56: 533-536.

25. Macrae WR, Owen M. Severe metabolic acidosis following hypotensive induced with sodium nitroprusside. Br J Anaesth 1974; 46: 795-797.

26. Merrified AJ, Blundell MD. Toxicity of sodium nitroprusside. Br J Anaesth 1974;46:795-797

27. Davies DW, Kadar D, Steward DJ, Munro IR. A sudden death associated with the use of sodium nitroprusside for induction of hypotensive during anaesthesia. Can Anaesth Soc J 1975; 46: 547-552.

28. Jack RD. Toxicity of sodium nitroprusside. Br J Anaesth 1974; 46: 952.

29. Prys-Roberts C, Milard RK. Self-tuning adaptive control of induced hypotensive in humans: a comparison of isoflurane and sodium nitroprusside. J Clin Monit 1990; 6:236-240.

30. Mankenzie AF, Colvin JR, Kenny GNC, Bisset WIK. Closed loop control of arterial hypotensive following intracranial surgery using sodium nitroprusside- A comparison of intraoperative halothane or isoflurance. Anaesthesia 1993:48:202-204

31. Gallagher DM, Milliken RA. Induced hypotensive for orthognathic surgery. J Oral Surgery 1979; 37:47-51.

32. Woodcock TE, Millard RK, Dixon J, Prys- Robers C. Clonidine premedication for isoflurane induced hypotension. Br J Anaesth 1988; 60:388-394.

33. Eichwede F, Komar K, Miller R, Grote B, Tarnow J. Preoperative treatment with $\beta$ blockers and isoflurane anesthesia. Hemodynamic interactions in patients with coronary disease Anaesthetist 1990; 39:361-366.

34. Bahlman SH, Eger EL II, Halsey MJ, Stevens WC, Shakespeare TF, Smith NT, Cromwell TH, Furcade $\mathrm{H}$. The cardiovascular effects of halothane in man during spontaneous ventilation. Anesthesiology 1972; 36:494-505.

35. Hunter AR. Neurosurgical Anaesthesia. Oxford, Black- Well Scientific Publications, 1996:147. 


\section{REVIEW ARTICLE}

36. Bernard JM, Quintin L, Plinaud M. Clonidine: from the treatment of hypertension to its use in anesthesia. II: perioperative use. Ann Fr Anesth Reanim 1990; 9:423-432.

37. Fridrich KL. Anesthetic techniques to reduce blood loss and transfer therapy. Oral Maxillofac Surg Clin North Am 1992; 4:863-864.

\section{AUTHORS:}

1. Baljit Singh Bajwa

\section{PARTICULARS OF CONTRIBUTORS:}

1. Assistant Professor, Department of Anaesthesia, Punjab Institute of Medical Sciences (PIMS), Jalandhar.

\section{NAME ADDRESS EMAIL ID OF THE} CORRESPONDING AUTHOR:

Dr. Baljit Singh Bajwa, Department of Anaesthesia and Critical Care,

Punjab Institute of Medical Sciences (PIMS), Garha Road, Jalandhar, Punjab.

Email: baljitbajwa7@gmail.com

Date of Submission: 14/07/2014. Date of Peer Review: 15/07/2014. Date of Acceptance: 21/07/2014. Date of Publishing: 24/07/2014. 